\title{
Correlations between Serum Levels of Interleukin-2 and Different Symptoms of Schizophrenic Disorders
}

M.M.El Hamady, A.M.Matta, Sh.T.El-Bakry, M.F.Ebrahim

Neuropsychiatry Dept., Faculty of Medicine, Benha Univ., Benha, Egypt

E-Mail: drm_egy21@yahoo.com

\begin{abstract}
Schizophrenia (SZ) is a chronic psychiatric disorder characterized by positive (e.g., hallucinations and delusions), negative (e.g., apathy and impaired motivation), and cognitive symptoms (e.g., poor performance in executive functions and attention). Interleukin-2( IL-2) has been one of the most frequently studied cytokines in schizophrenia. Several studies have pointed to a potential role of IL-2 in SZ, with most studies reporting altered peripheral levels of IL-2 when compared with healthy controls. The aim of this work is to determine serum level of interlukin-2 in a sample of schizophrenic patients. Also to compare the serum levels of IL-2 in schizophrenic patients and healthy controls and to investigate a possible correlation with positive and negative symptoms of. This analytic (case control) study included thirty patients with schizophrenia and a similar number of normal healthy individuals as control group. Both groups were subjected to a semi structured interview, SCID, PANSS and serum level of IL-2.
\end{abstract}

Keywords: Interleukin-2, Schizophrenia, Immunology.

\section{Introduction}

Schizophrenia is a serious and debilitating psychiatric disorder that affects nearly $1 \%$ of the world's population and is diagnosed by characteristic psychotic and non-psychotic symptoms [1].Positive symptoms involve impaired reality testing and include delusions and hallucinations [2]. While negative symptoms include blunted affect, alogia, asociality, anhedonia and avolation [3] To elucidatethe pathogenic mechanism of SZ, multiple hypotheses have been proposed such as neurodevelopmental hypothesis , dopamine hypothesis, glutamate hypothesis and cytokine imbalance hypothesis[4]. Interleukin-2 (IL-2) is a well-known cytokine that plays an important role in multiple immunoregulatory functions related to T-cells in peripheral and central nervous system(CNS)[5]. IL-2 is of particular interest to researchers because it has been shown to modulate some neurotransmitter systems including dopamine metabolism within the central

nervous system[6]. Numerous previous studies have shown contradictory results as some proved a decreased in vitro production of IL-2 in schizophrenia patients $[7,8,9]$ while others observed an increased IL2 levels in the serum, plasma and cerebrospinal fluid (CSF) of schizophrenia patients[10].

\section{Patients and methods}

This case control study included thirty patients with schizophrenia who were recruited from the Neuropsychiatry outpatient clinic of Benha University Hospital and inpatient and outpatient clinic of Benha Psychiatric Hospital. Thirty matched healthy individuals with same age, sex, social class as patients were added. The study was assessed and approved by the

institutional ethics committee. The procedure was explained to the patients and informed consent was obtained. All participants underwent a semistructured interview, Structured Clinical Interview for DSM Disorders (SCID I), positive and negative syndrome

scale (PANSS)for severity of psychotic symptoms, and serum interleukin-2(IL-2) measurement by ELISA. Exclusion criteria were age less than 18 years old, comorbid autoimmune disease or substance abuse, other neuropsychiatric or neurological disorders. All patients complaining of psychotic symptoms were examined for the validation of diagnosis of schizophrenia.This took place at the psychiatric clinic in Benha University Hospital and inpatient and outpatient clinic of Benha Psychiatric Hospital.A total number of 60 schizophrenic patients were examined.Comorbidity was present in 12 cases which was either substance abuse or psychiatric or neurological disorder.10 refused to participate due to long time of procedures or were hesitant regarding blood sample.Another 8 cases dropped out and did not follow up.

All cases and controls were subjected to a thorough clinical,psychiatric history and examination.They were given another appointment for the psychometric assessment and the blood sampling, usually it took more than one visit. Due to several visits and sometimes patients and relatives had to wait before the assessment was done, several cases and controls dropped out. 5 first degree relatives when assessed by SCID I were diagnosed by psychiatric disorders as depressive and anxiety disorders. Some patients and relatives were spectacle about giving a blood sample as they were worried or felt it was not necessary as it would not help in the cure of the patient .Further explanation about the aim of the research was rediscussed.

Finally,the 30 cases and 30 controls were examined ,assessed and a blood sample was received.

\section{Statistical Analysis}

In the statistical comparison between the different groups, the significance of difference was tested using one of the following tests:-

1-Student's t-test:- Used to compare mean of two groups of quantitative data, ANOVA test ( $F$ value) and kruskal-wallis test:-Used to compare mean of more than two groups of quantitative data of 
parametric and non-parametric respectively and, inter-group comparison of categorical data was performed by using chi square test $\left(\mathrm{X}^{2}\right.$-value) and fisher exact test (FET) and correlation coefficient:to find relationships between variables.

A $P$ value $<0.05$ was considered statistically significant $(*)$ while $>0.05$ statistically insignificant $\mathrm{P}$ value $<0.01$ was considered highly significant $(* *)$ in all analyses.

\section{Results}

The study enrolled 16 male and 14 female in case group with a mean age of $35.1 \pm 9.34$ years and 15 male and 15 female in control group with a mean age of 36.67 \pm 8.6 years Table (1).

Seven cases scored mild , 17 scored moderate, 3 scored severe and 2 scored very severe according to PANSS Table (2) .

There was no statistical difference among schizophrenic patients regarding mean serum level of IL-2 according to sociodemographic data Fig(1). Also, there was no statistical difference among schizophrenic patients regarding mean serum level of IL-2 according to clinical data but it was less in

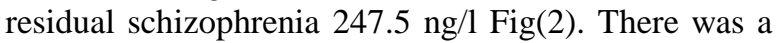
statistical significant difference $(0.04)$ regarding total score of PANSS and serum level of IL-2Table(3).

\section{Discussion}

In the last decade, immunological alterations in individuals affected by major mental disorders, such as SZ, have received great attention, as they aid in further elucidation of the related pathophysiological pathways. One of the most promising approaches to evaluate immune changes in SZ is the measurement of cytokines in serum or plasma. Cytokines are involved in a complex and redundant network that communicates immune and non immune cells[11].

The current study aimed at determining serum level of interlukin-2 in a sample of schizophrenic patients and to investigate a possible correlation of peripheral IL-2 levels with positive and negative symptoms of different schizophrenic disorders. Moreover, the study aimed at comparing healthy controls with schizophrenic patients to find out an association with peripheral IL-2 level. Thirty schizophrenic patients were recruited and matching healthy individuals with same age ,sex , social class as the sample of patients were also recruited. All participants were subjected to a semi-structured interview that emphasized some important factors i.e. socio demographic data including age, sex, residency, marital status and occupational state, A thorough general medical ,neurological examination and clinical psychiatric assessment was done. Also they were assessed by suitable psychometric test i.e. SCID1,PANSS, and serum Interlukin-2 measurement.

In the current study the ratio between male and female in schizophrenic patients was $53.3 \%: 46.7 \%$ i.e. 1.1:1.This is in agreement with findings of McGrath et al (2008) who studied the prevalence of schizophrenia in the general population and did not find gender differences[12]. There was a highly statistical significant difference ( $\mathrm{p}<0.001)$ between the schizophrenic patients and control group regarding marital status and occupation. About two thirds( $63.3 \%$ )of patients were single and $53.3 \%$ were unemployed which may be a consequence of the social and occupational deterioration associated with schizophrenia as the impairment of functional outcomes is a core problem in schizophrenia.

On the same line, estimated that , $65.7 \%$ were unemployed[13]. And on the other hand, in his study found that $37.1 \%$ of schizophrenic patients were unemployed [14]. while found that $12.1 \%$ were unemployed [15].

Regarding comparison between schizophrenic patients and control group regarding the four subscales of PANSS and its total score there was a highly statistical significance between both groups as this psychometry is used to diagnose schizophrenia while control group were healthy individuals.

Regarding severity of schizophrenia according to PANSS 1(3.3.\%),7(23.3\%), $17 \quad(56.7 \%), 3(10 \%)$ and $2(6.7 \%)$ scored negative, mild, moderate, severe and very severe respectively on total score. Regarding mean serum level of IL-2 there was no statistical significant difference between both groups.

In concomitant with this finding is what was estimated by in their meta-Analysis of cytokine alterations in schizophrenia who found no significant difference between SCZ patients and healthy controls regarding serum level of IL-2 [16].

Following the same trend, in their meta-analysis of blood cytokine network alterations in psychiatric patients: comparisons between schizophrenia, bipolar disorder and depression concluded that IL-2 level did not differ in chronically ill patients with schizophrenia versus controls[17].

In contrast, found lower levels of serum IL-2 in schizophrenic patients[18].

Likely contributing factors to this disarray include studies sampling patients at different stage of illness, different illness course, acutely unwell or in remission. Differences in age, gender, smoking, body mass index (BMI), and recent infections will also have significant impact yet are not controlled for[19].

In addition, the different essay method, stored period of the sample, differences in tested material (serum vs plasma),exposure to a variety of different dosage and duration of antipsychotic treatments, subtypes of schizophrenic patients recruited. Also the inconsistent results may be due to the fact that schizophrenia is generally treated as though it were a single disease process, instead of several etiologically distinct disorders.

Regarding socio demographic data there were no significant differences in serum level of IL-2 among case group either as regards sex, marital status, occupation or smoking. Compiling with the results of 
the current study, noted that within the patient group, no significant correlation between IL-2 levels and any demographic parameters[20].

Regarding serum levels of interleukin-2 in the case group according to clinical data of schizophrenia. The findings revealed no significant difference among case group regarding type of schizophrenia.In consistent with these findings is what was estimated by who found no significant effects of schizophrenia subtype on IL-2[20].

There was a statistical significant difference (0.04) regarding total score of PANSS and serum level of IL2.On the same line, found a significant positive relationship between sIL-2r levels and total PANSS scores implying that elevated serum levels of sIL-2r somehow may be related to greater psychopathology[21].

Table (1) Differences between case and control group according to sociodemographic data.

\begin{tabular}{|c|c|c|c|c|c|c|}
\hline & \multicolumn{2}{|c|}{ Case group (30) } & \multicolumn{2}{|c|}{ Control group (30) } & \multirow{2}{*}{$\begin{array}{c}\text { Statistical } \\
\text { test }\end{array}$} & \multirow{2}{*}{ P value } \\
\hline & No & $\%$ & No & $\%$ & & \\
\hline \multicolumn{7}{|l|}{ Sex } \\
\hline Male & 16 & 53.3 & 15 & 50.0 & $X^{2}=0.07$ & 0.80 \\
\hline Female & 14 & 46.7 & 15 & 50.0 & & \\
\hline mean \pm SD & \multicolumn{2}{|c|}{$35.1 \pm 9.34$} & \multicolumn{2}{|c|}{$36.67 \pm 8.6$} & St $\mathrm{t}=0.68$ & 0.50 \\
\hline \multicolumn{7}{|l|}{ Marital status } \\
\hline Single & 19 & 63.3 & 4 & 13.3 & \multirow{4}{*}{$\mathrm{FET}=22.34$} & \multirow{4}{*}{$<0.001 * *$} \\
\hline Married & 7 & 23.3 & 23 & 76.7 & & \\
\hline Divorced & 3 & 10.0 & 0 & 0.0 & & \\
\hline Widowed & 1 & 3.3 & 3 & 10.0 & & \\
\hline \multicolumn{7}{|l|}{ Occupation } \\
\hline No & 16 & 53.3 & 0 & 0.0 & \multirow{2}{*}{$X^{2}=21.82$} & \multirow[t]{2}{*}{$<0.001 * *$} \\
\hline Yes & 14 & 46.7 & 30 & 100 & & \\
\hline \multicolumn{7}{|l|}{ Occupation } \\
\hline Unemployed & 16 & 53.3 & 0 & 0.0 & \multirow{6}{*}{$\mathrm{FET}=28.69$} & \multirow{6}{*}{$<0.001 * *$} \\
\hline Student & 2 & 6.7 & 2 & 6.7 & & \\
\hline Manual worker & 5 & 16.7 & 7 & 23.3 & & \\
\hline Higher performance & 1 & 3.3 & 6 & 20.0 & & \\
\hline Housewife & 6 & 20.0 & 10 & 33.3 & & \\
\hline Farmer & 0 & 0.0 & 5 & 16.7 & & \\
\hline \multicolumn{7}{|l|}{ Smoking } \\
\hline Yes & 17 & 56.7 & 11 & 36.7 & \multirow{2}{*}{$X^{2}=2.41$} & \multirow[t]{2}{*}{0.12} \\
\hline No & 13 & 43.3 & 19 & 63.3 & & \\
\hline
\end{tabular}

** Highly significant

Table (2) Differences between case and control group according to PANSS.

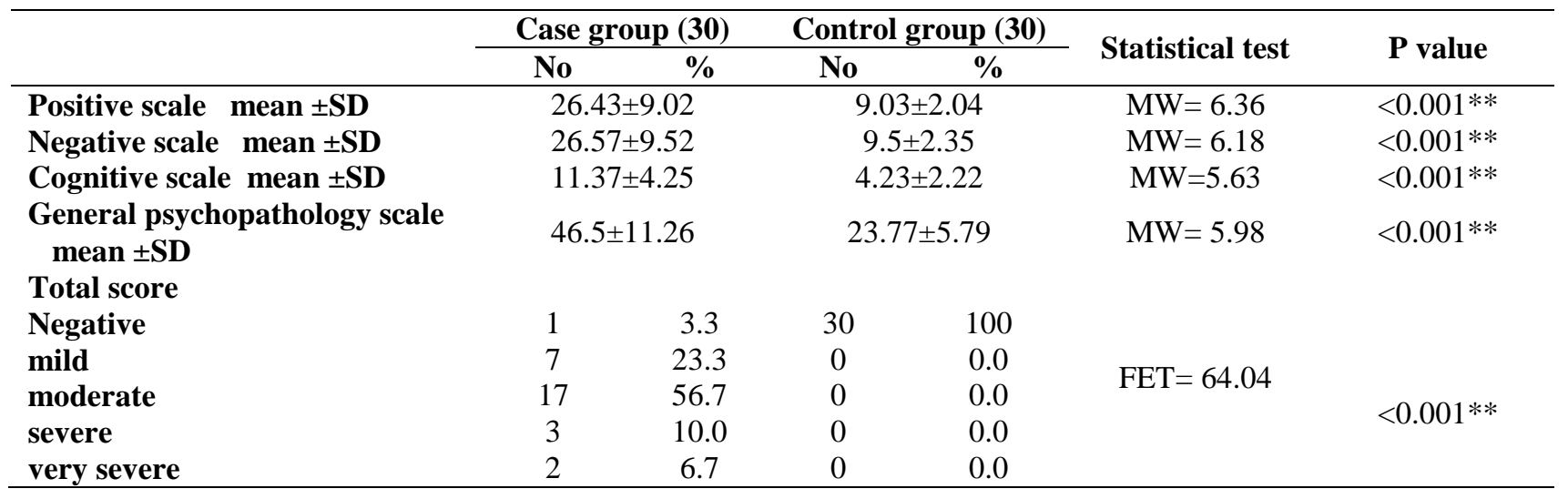

** Highly significant 


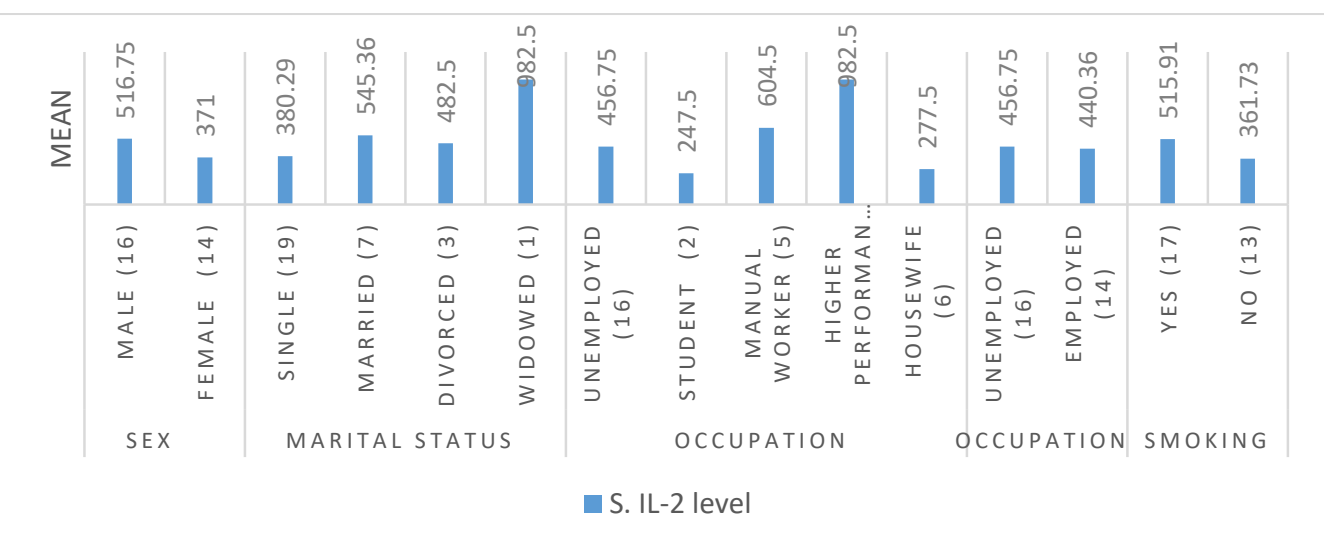

Fig (1) Mean level of serum IL-2 in case group according to socio-demographic data.

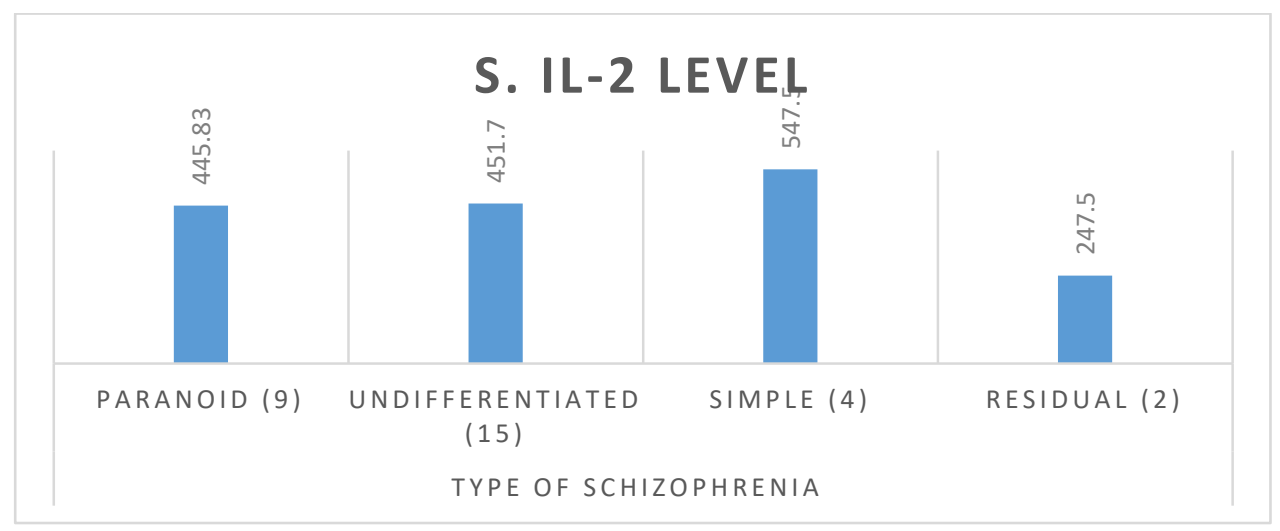

Fig (2) Mean level of serum IL-2 in case group according to types of schizophrenia

Table(3) Serum IL-2 level and different variables among case group according to total score of PANSS.

\begin{tabular}{lccccc}
\hline Case group (30) & \multicolumn{3}{c}{ S. IL-2 level } & \multirow{2}{*}{ Statistical test } & \multirow{2}{*}{ P value } \\
\cline { 2 - 4 } & Mean & $\mathbf{\pm S D}$ & Range & & \\
Total score of & & & & \\
PANSS & 982.5 & - & $982.5-982.5$ & \multirow{2}{*}{$\mathrm{F}=2.95$} & $0.04 *$ \\
Negative (1) & 613.93 & 274.25 & $142.5-982.5$ & & \\
Mild (7) & 341.21 & 212.63 & $40.5-742.5$ & \\
Moderate (17) & 532.5 & 392.3 & $82.5-802.5$ & \\
Severe (3) & 397.5 & 21.21 & $382.5-412.5$ & & \\
Very severe (2) & &
\end{tabular}

*Significant

\section{Conclusion}

This study concludes that no statistical significant difference between case and control group regarding serum level of IL-2 while there was a statistical significance between serum level of interleukin-2 and total score of PANSS. IL-2 might get involved in the severity of psychopathological state, suggesting that IL-2 seems to be a promising molecular biomarker for further investigations in severity of schizophrenia.

\section{References}

[1] M.Green, W.Horan, J.Lee,Social cognition in schizophrenia, Nat Rev Neurosci ,vol.16, pp.620$631,2015$.

[2] K. HÄNNINEN, Selected Genetic Polymorphisms of Epidermal Growth Factor, Dopamine-2 Receptor, Neuregulin-1 and Inflammatory Cytokines in Schizophrenia. Academic dissertation. School of Medicine of the University of Tampere, Vol.2 (5),pp.15-23,2014.

[3] S. Galderisi, A.Mucci , R.W.Buchanan, Arango, Negative symptoms of schizophrenia: new 
developments and unanswered research questions, The lancet psychiatry, Vol.5(8), PP.664677,2011 .

[4] L .Gao, Z. Li, S .Chang, J .Wang ,AssociationofInterleukin-10 Polymorphisms with Schizophrenia: A Meta-Analysis. PLoS ONE, Vol.9(3), PP.e90407.10.1371/ journal. pone.0090407,2014.

[5] E.G.deAraujo, G. M.da Silva, A.A.dos Santos "Neuronal cell survival: the role of interleukins," Annals of the New York Academy of Sciences, Vol.1153, pp. 57-64,2009.

[6]J.M.Petitto, D. Meola, Z.Huang, Interleukin-2 and the brain: dissecting central versus peripheral contributions using unique mouse models. Methods Mol. Biol, Vol.934, pp.301-311,2012.

[7] R.M.Craddock, H.E. Lockstone, D.A.Rider, M.T.Wayland, L.J.Harris, P.J.Mc Kenna, S.Bahn, Altered T-cell function in schizophrenia: a cellular model to investigate molecular disease mechanisms. PLoS One, Vol.2 (8), PP.e692,2007.

[8] S. Potvin, E. Stip, A.A. Sepehry, Inflammatory cytokine alterations in schizophrenia: A systematic quantitative review. Biol Psychiatry 63,pp.801-808,2008. , Vol.

[9] J.Guo, C.Liu, W. Y.ang, B.Feng, X.Zhang, Role of $\mathrm{T}$ helper lymphokines in the immuneinflammatory pathophysiology of schizophrenia: systematic review and meta-analysis. Nord. J. Psychiatry ;vol;69 (5), pp.364-372,2015.

[10] H.M.An, Y.L.Tan, J.Shi, Z.R.Wang, J.C.Soars, J.Q.Wu, F.D.Yang, X.F.Huang, X.Y.Zhang, Altered IL-2, IL-6 and IL-8 serum levels in schizophrenia patients with tardive dyskinesia. Schizophr. Res, Vol.162 (1-3), pp.261-268,2015.

[11] E.Asevedo, L.B.Rizzo, A.Gadelh, R.B.Mansur, V.K.Ota, A.A.Berberian, B.S.Scarpato, A.L.Teixeira, , R.A.Bressan, , E.Brietzke, Peripheral interleukin-2 level is associated with negative symptoms and cognitive performance in schizophrenia. Physiol. Behav, Vol.129, PP.194$198,2014$.

[12] J.McGrath, S.Saha, D.Chant, J.Welham "Schizophrenia: a concise overview of incidence, prevalence, and mortality," Epidemiologic Reviews, Vol.30(1),pp.67-76, 2008.
[13] A.U“cok, P.Gorwood, G.Karaday1, on behalf of the EGOFORS, Employment and its relationship with functionality and quality of life in patients with schizophrenia: EGOFORS Study. European Psychiatry, Vol.27,pp.422-425,2011.

[14] Y.T.Xiang , C.Y.Wang, Y.Wang, H.F .Chiu, J.P.Zhao, Q.Chen , Socio-demographic and clinical determinants of quality of life in Chinese patients with schizophrenia: a prospective study. Qual Life Res, Vol.19,pp.317-322,2010.

[15] M.Brink, A.Green, A.B.Bojesen, Excess medical comorbidity and mortality across the lifespan in schizophrenia., Schizophrenia Research, Vol.206,pp.347-354,2019.

[16] B.J.Miller, P.Buckley, W.Seabolt, A.Mellor, B.Kirkpatrick ,Meta-Analysis of Cytokine Alterations in Schizophrenia: Clinical Status and Antipsychotic Effects, BIOL PSYCHIATRY, Vol.70,pp.663-671,2011.

[17] D.R.Goldsmith, M.H.Rapaport , B.J. Miller ,A meta-analysis of blood cytokine network alterations in psychiatric patients: comparisons between schizophrenia, bipolar disorder and depression. Molecular Psychiatry , Vol.21,pp.1696-1709,2006.

[18] B.Singh , N.K.Bera, C.R.Nayak, T.K. Chaudhuri, Decreased serum levels of interleukin2 and interleukin-6 in Indian Bengale schizophrenic patients. Cytokine , Vol.47,pp.15,2009 .

[19] R. Upthegrove, N.M.Barnes, Immune system and schizophrenia: and update for clinicians. Adv. Psychiatr. Treat, Vol.20 (2), PP.83-91,2014.

[20] Y.Tan, Y.Li, S.Tan, Z.Wang, F. D.Yang, , B.Cao, G.B.Zunta-Soares, J.C.Soares, X. Y.Zhang, Increased interleukin-2 serum levels were associated with psycho-pathological symptoms and cognitive deficits in treatmentresistant schizophrenia. Schizophr. Res, Vol.169, PP.16-20,2015.

[21] C.Bresee , M.H.Rapaport ,Persistently increased serum soluble interleukin-2 receptors in continuously ill patients with schizophrenia. International Journal of Neuropsychopharmacology , Vol.12,pp.861865,2009 . 